\title{
ON THE INVERSE OF MAÑÉ'S PROJECTION
}

\author{
H. MOVAHEDI-LANKARANI
}

(Communicated by James E. West)

\begin{abstract}
We show that every compact ultrametric space is bi-Lipschitz embeddable in a Hilbert space. We also provide an example of a compact ultrametric space whose fractal (and hence Hausdorff) dimension is finite, but which cannot be bi-Lipschitz embedded in any finite dimensional Euclidean space. This example, in particular, establishes that the inverse of Mañé's projection need not be Lipschitz even in the case of finite fractal dimension.
\end{abstract}

\section{INTRODUCTION}

In [3] Mañé proved that if $M$ is a compact subset of a Banach space $\mathscr{B}$ with the Hausdorff dimension of $M$ less than or equal to the integer $n$, then a residual set of continuous projections $P$ of $\mathscr{B}$ onto $(2 n+1)$-dimensional linear subspaces are injective on $M$. Recently, Eden, et. al. [2] have noted that it is not known, in general, whether the inverse map $\left(\left.P\right|_{M}\right)^{-1}$ satisfies further continuity properties. They do establish that if $M$ is a compact subset of $\mathbb{R}^{N}$ such that the fractal dimension of $M$ is no greater than the integral part of $\frac{1}{2}(N-1)$, then the inverse of Mañé's projection $\left(\left.P\right|_{M}\right)^{-1}$ is Hölder continuous of order $\theta$ for any $\theta$ sufficiently small. They also provide an example of a compact subset $M$ of a Hilbert space with the property that the Hausdorff dimension of $M$ is finite (in fact zero), but $\left(\left.P\right|_{M}\right)^{-1}$ is not Hölder continuous of any order. This space $M$, however, has infinite fractal dimension and one may hope that the situation is different for sets of finite fractal dimension.

In this note we construct a compact subset $M$ of an infinite dimensional Hilbert space $\mathscr{H}$ such that the fractal dimension of $M$ is finite (less than or equal to 4), but $M$ is not bi-Lipschitz embeddable in any finite-dimensional Euclidean space. This fact in turn implies that for any projection $P$ of finite rank, $\left(\left.P\right|_{M}\right)^{-1}$ is not even locally Lipschitz. These disagreeable properties follow from the fact that $M$ is not "really" finite-dimensional. That is, with respect to a more delicate notion of dimension, $M$ is infinite-dimensional. This dimension is the metric dimension, a refinement of the dimension introduced by Assouad [1], generalizing the dimensional order of Bouligand (1928).

In $\S 2$ we present a new proof that every compact ultrametric space is biLipschitz embeddable in a Hilbert space. We note here that this theorem follows

Received by the editors March 8, 1991.

1991 Mathematics Subject Classification. Primary 54E35, 46C05, 54E40. 
from a bi-Lipschitz embedding result of Assouad [1]. However, a refinement of our method of proof produces minimal bi-Lipschitz embeddings of compact ultrametric spaces of finite metric dimension in finite dimensional Euclidean spaces [4]. In $\S 3$ we construct an abstract ultrametric space whose fractal dimension is finite, but whose metric dimension is infinite. To get the desired counterexample, we then bi-Lipschitz embed this abstract space into a Hilbert space.

\section{BASIC MACHINERY}

In this section we prove a bi-Lipschitz embedding theorem for compact ultrametric spaces. A metric space $(M, d)$ is an ultrametric space if the metric satisfies a stronger form of the triangle inequality

$$
d(x, y) \leq \max \{d(x, z), d(y, z)\}
$$

for all $x, y, z \in M$. One special property of these spaces is the fact that any two closed balls in an ultrametric space are either disjoint or one is contained in the other. In particular, if two closed balls have the same radius, then they are either disjoint or equal. Consequently, every ultrametric space is totally disconnected. Furthermore, if $M$ is a countable product of countable factors, one may construct an ultrametric for $M$ in a way similar to the decimal expansion of real numbers. Specifically, let $\left\{r_{n}\right\}_{n \geq 1} \subset(0, \infty)$ be a strictly decreasing sequence converging to zero. For $x=\left(x_{k}\right)_{k \geq 1}$ and $y=\left(y_{k}\right)_{k \geq 1}$ in $M$, set $d(x, y)=r_{n}$, where $x_{k}=y_{k}$ for $1 \leq k \leq n-1$ and $x_{n} \neq y_{n}$. The following proposition is a converse to this observation.

Proposition 2.1. Every separable ultrametric space $(M, d)$ is bi-Lipschitz isomorphic to an inverse limit in a countable product of countable factors.

Proof. Let $\left\{r_{n}\right\}_{n \geq 1} \subset(0, \infty)$ be such that

(a) $\lim _{n \rightarrow \infty} r_{n}=0$;

(b) There is $0<K<\infty$ such that $1<r_{n-1} / r_{n} \leq K, \forall n \geq 1$.

Construct a sequence $\left\{\mathscr{F}_{n}\right\}_{n=1}^{\infty}$ of countable covers of $M$ by setting $\mathscr{F}_{n}=$ $\left\{B\left(x, r_{n}\right) \mid x \in M\right\}$, where $B\left(x, r_{n}\right)=\left\{y \mid d(x, y) \leq r_{n}\right\}$. It follows that for each $n \in \mathbb{N}$ the cover $\mathscr{F}_{n}$ is a disjoint cover of $M$. Let $g_{n}: \mathscr{F}_{n+1} \rightarrow \mathscr{F}_{n}$ by setting

$$
g_{n}\left(B\left(\cdot, r_{n+1}\right)\right)=\text { the } r_{n} \text {-ball that contains } B\left(\cdot, r_{n+1}\right) \text {, }
$$

and let

$$
\Sigma=\lim \left(\mathscr{F}_{1} \stackrel{g_{1}}{\leftarrow} \mathscr{F}_{2} \stackrel{g_{2}}{\leftarrow} \mathscr{F}_{3} \stackrel{g_{3}}{\leftarrow} \cdots\right)=\lim \left(\mathscr{F}_{n}, g_{n}\right) .
$$

Define the map $g: M \rightarrow \Sigma$ by setting

$$
g(x)=\left(B\left(x, r_{1}\right), B\left(x, r_{2}\right), B\left(x, r_{3}\right), \ldots\right)=\left\{B\left(x, r_{n}\right)\right\}_{n} .
$$

Give $\Sigma$ the ultrametric $\rho$ defined by the sequence $\left\{r_{n}\right\}_{n=1}^{\infty}$. Specifically, $\rho\left(B, B^{\prime}\right)=r_{n}$ whenever the balls $B_{i}=B_{i}^{\prime}$ for $i \leq n-1$ but $B_{n} \neq B_{n}^{\prime}$.

Now, if $x, y \in M$ are in the same $r_{n-1}$-ball but in distinct $r_{n}$-balls, then $r_{n}<d(x, y) \leq r_{n-1}$. Then

$$
\rho(g(x), g(y)) \leq d(x, y) \leq K \rho(g(x), g(y)) .
$$

Consequently, the map $g: M \rightarrow \Sigma$ is a bi-Lipschitz isomorphism. 
As an immediate corollary of this proposition we see that a compact, perfect, ultrametric space is bi-Lipschitz equivalent to a Cantor space, that is, to a metric space homeomorphic to the Cantor set. The converse of this statement is false, however, not every Cantor metric space is bi-Lipschitz equivalent to an ultrametric space [4].

We are interested in bi-Lipschitz embeddings of a compact ultrametric space $(M, d)$ in a Hilbert space $\mathscr{H}$. Clearly, if $\mathscr{H}$ is an infinite-dimensional Hilbert space, then there exists $0<\varepsilon_{o}<1$ such that the open unit ball of $\mathscr{H}$ contains infinitely many disjoint closed $\varepsilon_{o}$-balls. Hence, we have the following:

Lemma 2.2. Let $0<r<\varepsilon_{o} / 2$. Then any open ball of radius $r^{n}, n \geq 1$, in $\mathscr{H}$ contains infinitely many disjoint closed balls of radius $2 r^{n+1}$.

Theorem 2.3. Let $\mathscr{H}$ be an infinite-dimensional Hilbert space and let $(M, d)$ be a compact ultrametric space. Then there is a bi-Lipschitz map $f: M \hookrightarrow \mathscr{H}$.

Proof. Let $0<r<\varepsilon_{o} / 2$ and let $r_{n}=r^{n}$. Then, by Proposition 2.1, $(M, d)$ is bi-Lipschitz isomorphic to $\Sigma=\lim \left(\mathscr{F}_{n}, g_{n}\right)$, where $\mathscr{F}_{n}$ is the family of closed disjoint $r^{n}$-balls and $g_{n}: \mathscr{F}_{n+1} \hookrightarrow \mathscr{F}_{n}$ is the natural inclusion characterized by

$$
g_{n}(B)=\text { the ball of } \mathscr{F}_{n} \text { containing } B \text {. }
$$

Because $(M, d)$ is compact, $\mathscr{F}_{n}$ is a finite set for each $n \geq 1$. Let $k_{n}$ denote the cardinality of $\mathscr{F}_{n}, n \geq 1$. We will show that there is a bi-Lipschitz embedding $\varphi: \Sigma \rightarrow \mathscr{H}$. We may construct a (nested) sequence $\left\{\mathscr{B}_{n}\right\}_{n \geq 1}$ of families of disjoint closed balls in $\mathscr{H}$ together with bijections $\nu_{n}: \mathscr{F}_{n} \rightarrow \mathscr{B}_{n}, n \geq 1$, as follows.

In the open unit ball of $\mathscr{H}$ choose a family $\widetilde{\mathscr{B}}_{1}$ of $k_{1}$ disjoint closed balls of radius $2 r$. Let $\mathscr{B}_{1}$ be the family of concentric closed balls of radius $r$ and choose a bijection $\nu_{1}: \mathscr{F}_{1} \rightarrow \mathscr{B}_{1}$. In the interior of each ball $\nu_{1}(B)$ of $\mathscr{B}_{1}$ we choose (by Lemma 2.2) a family $\widetilde{\mathscr{B}}_{2}^{B}$ of $\operatorname{card}\left(g_{1}^{-1}(B)\right)$ disjoint closed balls of radius $2 r^{2}$. Let $\mathscr{B}_{2}^{B}$ be the family of concentric closed balls of radius $r^{2}$ and choose a bijection $\nu_{2}^{B}: g_{2}^{-1}(B) \rightarrow \mathscr{B}_{2}^{B}$. Let $\mathscr{B}_{2}=\bigcup\left\{\mathscr{B}_{2}{ }_{2} \mid B \in \mathscr{F}_{1}\right\}$ and define a bijection $\nu_{2}: \mathscr{F}_{2} \rightarrow \mathscr{B}_{2}$ by setting $\nu_{2}(C)=\nu_{2}^{B}(C)$ when $C \subset B$. Then the diagram

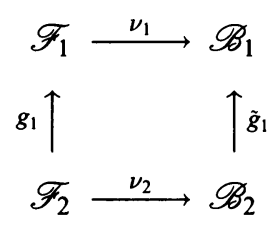

commutes, where $\tilde{g}_{1}$ is the natural map induced by inclusion.

Suppose inductively that $\mathscr{B}_{k}$ and $\nu_{k}: \mathscr{F}_{k} \rightarrow \mathscr{B}_{k}$ have been constructed for $k \leq n$. Then we have the diagram

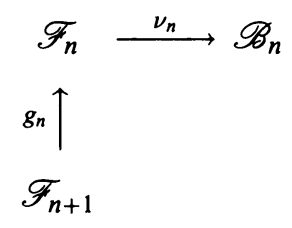


For each $B \in \mathscr{F}_{n}$ we choose (by Lemma 2.2) a family $\widetilde{\mathscr{B}}_{n+1}^{B}$ of $\operatorname{card}\left(g_{n}^{-1}(B)\right)$ disjoint closed balls of radius $2 r^{n+1}$ in the interior of $\nu_{n}(B)$. Let $\mathscr{B}_{n+1}^{B}$ be the concentric balls of radius $r^{n+1}$ and choose a bijection $\nu_{n+1}^{B}: g_{n}^{-1}(B) \rightarrow \mathscr{B}_{n+1}^{B}$. Let $\mathscr{B}_{n+1}=\bigcup\left\{\mathscr{B}_{n+1}^{B} \mid B \in \mathscr{F}_{n}\right\}$ and define a bijection $\nu_{n+1}: \mathscr{F}_{n+1} \rightarrow \mathscr{B}_{n+1}$ by setting $\nu_{n+1}(C)=\nu_{n+1}^{B}(C)$ when $C \subset B$. Then the diagram

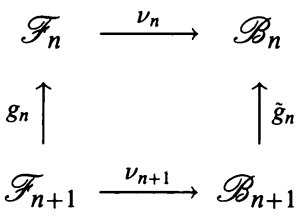

commutes, where $\tilde{g}_{n}$ is the natural map induced by inclusion; and the induction step is complete.

Now, define $\varphi: \Sigma \rightarrow \mathscr{H}$ by setting

$$
\{\varphi(x)\}=\bigcap_{n=1}^{\infty} \nu_{n}\left(x_{n}\right),
$$

where $x=\left(x_{n}\right)_{n} \in \Sigma$. If $x, y \in \Sigma$ with $\rho(x, y)=r^{n}$ for some $n$, then $x_{i}=y_{i}$ for $1 \leq i \leq n-1$ and $x_{n} \neq y_{n}$. Hence, $\varphi(x)$ and $\varphi(y)$ are in the same member of $\mathscr{B}_{n}$ but in different members of $\mathscr{B}_{n+1}$. Therefore,

$$
2 r^{n+1} \leq\|\varphi(x)-\varphi(y)\|_{\mathscr{R}} \leq r^{n},
$$

which gives

$$
\varepsilon_{o} \rho(x, y)<\|\varphi(x)-\varphi(y)\| \mathscr{H} \leq \rho(x, y) .
$$

Consequently, the map $\varphi: \Sigma \hookrightarrow \mathscr{H}$ is a bi-Lipschitz embedding. Finally, we let $f: M \hookrightarrow \mathscr{H}$ by setting $f(x)=(\varphi \circ g)(x), x \in M$. Clearly, $f$ is a bi-Lipschitz map since the maps $g: M \rightarrow \Sigma$ and $\varphi: \Sigma \hookrightarrow \mathscr{H}$ are bi-Lipschitz.

One may use the metric dimension (defined below) to obtain a refinement of this theorem. Specifically, if $(M, d)$ is compact ultrametric space of metric dimension $D$, then there is a bi-Lipschitz embedding of $(M, d)$ in $\mathbb{R}^{N}$ for any $N>D$. (A proof will appear elsewhere.)

\section{THE EXAMPLE}

Before we construct our counterexample, we recall that the (upper) fractal dimension of a compact metric space $(M, d)$ is defined by the limit

$$
\operatorname{dim}_{f}(M)=\lim _{r \rightarrow 0} \sup \frac{\log N_{r}(M)}{\log (1 / r)},
$$

where $N_{r}(M)$ denotes the minimum number of open $r$-balls necessary to cover $M$. However, we will utilize the metric dimension of $(M, d)$, denoted by $\operatorname{dim}_{m}(M)$, defined as follows.

Definition 3.1. Let $(M, d)$ be a metric space and for $0<r_{2}<r_{1}$ let $\mathscr{N}\left(r_{1}, r_{2}\right)$ denote the maximum number of disjoint closed $r_{2}$-balls contained in a closed $r_{1}$-ball. Let

$$
\Delta_{r, t}(M)=\sup \left\{\frac{\log \mathscr{N}\left(r_{1}, r_{2}\right)}{\log \left(r_{1} / r_{2}\right)} \mid 0<r_{2}<r_{1}<r \text { and } r_{1}>t r_{2}\right\} .
$$


Then the metric dimension of $M$ is defined by

$$
\operatorname{dim}_{m}(M)=\lim _{r \rightarrow 0} \lim _{t \rightarrow \infty} \Delta_{r, t}(M) .
$$

These limits exist since they are monatonic, but may be infinite.

The metric dimension satisfies all the usual properties that a reasonable dimension should satisfy. Specifically,

(i) If $\left(M_{1}, d_{1}\right)$ is a metric subspace of $\left(M_{2}, d_{2}\right)$, then $\operatorname{dim}_{m}\left(M_{1}\right)$ $\leq \operatorname{dim}_{m}\left(M_{2}\right)$.

(ii) If $M$ is an open subset of $\mathbb{R}^{n}$ equipped with the inherited metric, then $\operatorname{dim}_{m}(M)=n$.

(iii) If $\left(M_{1}, d_{1}\right)$ and $\left(M_{2}, d_{2}\right)$ are bi-Lipschitz isomorphic, then $\operatorname{dim}_{m}\left(M_{1}\right)=\operatorname{dim}_{m}\left(M_{2}\right)$.

The metric dimension is indeed different from the fractal dimension (as well as the Hausdorff dimension), since our example has finite (upper) fractal dimension but infinite metric dimension.

Example 3.2. Let $q_{1}=2$ and $q_{n}=q_{1} \cdots q_{n-1}+1, n \geq 1$. Let $r_{1}=1 / 2$ and for $n \geq 1$ let $r_{2 n}=\left(q_{1} \cdots q_{2 n-1}\right)^{-1}$ and $r_{2 n+1}=\frac{1}{n} r_{2 n}$. Let $\mathbb{Z}_{q_{n}}=\mathbb{Z} / q_{n} \mathbb{Z}$ and set $M=\prod_{n \geq 1} \mathbb{Z}_{q_{n}}$. For $x=\left(x_{i}\right)_{i \geq 1}$ and $y=\left(y_{i}\right)_{i \geq 1}$ in $M$ let $d(x, y)=r_{n}$ if $x_{i}=y_{i}$ for $1 \leq i \leq n-1$ and $x_{n} \neq y_{n}$. It is clear the $d$ is an ultrametric on $M$ and that $(M, d)$ is compact. We first compute the fractal dimension of $M$. To this end, note that if $r_{n}<r \leq r_{n-1}$, then

$$
\begin{aligned}
N(r) & =\text { number of open } r \text {-balls needed to cover } M \\
& =\text { number of closed } r_{n} \text {-balls needed to cover } M \\
& =q_{1} \cdot q_{2} \cdots \cdots q_{n-1} .
\end{aligned}
$$

Therefore, if $n$ is even, then

$$
\frac{\log N(r)}{\log (1 / r)}<1+\frac{\log 8+3 \log \left(q_{1} \cdots q_{2 n-3}\right)}{\log \left(q_{1} \cdots q_{2 n-3}\right)}
$$

and if $n$ is odd, then

$$
\frac{\log N(r)}{\log (1 / r)} \leq 1+\frac{\log \left(q_{1} \cdots q_{2 n-1}+1\right)}{\log \left(q_{1} \cdots q_{2 n-1}\right)} .
$$

Consequently, $\operatorname{dim}_{f}(M) \leq 4$.

However, for any $n \geq 1$ we have

$$
\frac{\log \mathcal{N}\left(r_{2 n}, r_{2 n+1}\right)}{\log \left(r_{2 n} / r_{2 n+1}\right)}=\frac{\log q_{2 n}}{\log n}
$$

which tends to infinity as $n$ tends to infinity. Hence, $\operatorname{dim}_{m}(M)=\infty$.

Next, let $\mathscr{H}$ be an infinite-dimensional Hilbert space. By Theorem 2.3, there is a bi-Lipschitz embedding $f: M \hookrightarrow \mathscr{H}$. Let $X=f(M)$. Since both the fractal dimension and the metric dimension are invariant under bi-Lipschitz maps, we see immediately that $\operatorname{dim}_{f}(X) \leq 4$ and $\operatorname{dim}_{m}(X)=\infty$. Hence, $X$ is a compact finite fractal dimensional subset of $\mathscr{H}$ that is not bi-Lipschitz embeddable (not even locally) in any finite-dimensional linear sutspace. In particular, if $P$ is any projection on $\mathscr{H}$ of finite rank, then $\left(\left.P\right|_{X}\right)^{-1}$ cannot 
be Lipschitz; thus providing a counterexample to Lipschitz continuity for the inverse of Mañé's projection.

We remark here that we do not know whether $\left(\left.P\right|_{X}\right)^{-1}$ is Hölder continuous of order $\alpha \neq 1$. The reason is that we do not know how the metric dimension behaves under Hölder maps. More specifically, if $M$ and $N$ are metric spaces and $f: M \rightarrow N$ is Hölder continuous of order $\alpha$ above and below, then $\operatorname{dim}_{m}(M)=\operatorname{dim}_{m}(N)$. However, if $f$ is Hölder continuous of order $\alpha$ above and $\beta$ below with $\alpha \neq \beta$, then it is not known how $\operatorname{dim}_{m}(M)$ and $\operatorname{dim}_{m}(N)$ are related.

\section{REFERENCES}

1. P. Assouad, Plongements Lipschitziens dans $\mathbb{R}^{n}$, Bull. Soc. Math. France 3 (1983), 429-448.

2. A. Eden, C. Foias, B. Nicolaenko, and R. Temam, Hölder continuity for the inverse of Mãné's projection, September 1990, IMA Preprint Series No. 695, University of Minnesota, Minneapolis, Minnesota 55455.

3. R. Mañé, Lecture Notes in Math., vol. 898, Springer-Verlag, New York, 1981, pp. 230-242.

4. H. Movahedi-Lankarani, Minimal Lipschitz embeddings, Ph.D. Thesis, Pennsylvania State Univ., 1990.

Department of Mathematics, Penn State Altoona Campus, Altoona, Pennsylvania $16601-3760$ 